\title{
Curiosity, latent learning, and cognitive maps
}

Department of Neuroscience, Center for Magnetic Resonance Research, and Center for Neuroengineering

University of Minnesota, Minneapolis MN 55455

\section{Corresponding author:}

Maya Zhe Wang

Department of Neuroscience and Center for Magnetic Resonance Research

University of Minnesota, Minneapolis MN 55455

Email: mayawangz@gmail.com

\section{Funding statement}

This research was supported by a National Institute on Drug Abuse Grant R01 DA038106 (to BYH).

\section{Competing interests}

The authors have no competing interests to declare.

\section{Acknowledgements}

We thank Ethan Bromberg-Martin for helpful discussions. 


\section{ABSTRACT}

37 Curiosity refers to a desire for information that is not driven by immediate strategic or

38 instrumental concerns. Latent earning refers to a form of learning that is not directly driven by

39 standard reinforcement learning processes. We propose that curiosity serves the purpose of

40 motivating latent learning. Thus, while latent learning is often treated as an incidental or passive

41 process, in practice it most often reflects a strong evolved pressure to consume large amounts of

42 information. That large volume of information in turn allows curious decision makers to generate

43 sophisticated representations of the structure of their environment, known as cognitive maps.

44 Cognitive maps facilitate adaptive and flexible behavior while maintaining its adaptivity and

45 flexibility via map updates based on new information. Here we describe data supporting the idea

46 that orbitofrontal cortex (OFC) and dorsal anterior cingulate cortex (dACC) play complementary

47 roles in curiosity-driven learning. Specifically, we propose that (1) OFC tracks the innate value

48 of information and incorporates new information into a detailed cognitive map; and (2) dACC

49 tracks the environmental demands and information availability to then use the cognitive map for

50 guiding behavior. 


\section{Introduction}

The natural environment offers a plethora of rewards to most foragers but acquiring these

53 rewards requires knowledge - for example, trees with ripe apples may be inhomogeneously

54 distributed in a forest (Calhoun and Hayden, 2015; Gottlieb and Oudeyer, 2018; Mobbs et al.,

55 2018; Oudeyer and Smith, 2016). Consequently, natural decision-makers are faced with a

56 constant overwhelming poverty of information. Any steps they can take to alleviate that poverty,

57 even fractionally, offers great survival benefits relative to rivals. This information gap is an area

58 where psychological and neuroscientific tasks used in the laboratory differ from those that occur

59 in the natural environment and that presumably drove our evolution. In the laboratory, research

60 subjects (both human and non-human) are typically given all information except that which is

61 experimentally relevant. As a consequence, there are areas where failure to consider the paucity

62 of valuable in our evolved environment can mislead us.

63 One of those areas is curiosity. Curiosity, which we can define as a drive for non-

64 strategic information, has long fascinated psychologists (Berlyne, 1966; Oudeyer et al., 2016;

65 Kidd and Hayden, 2015; Byrne, 2013; Loewenstein, 1994; Golman and Loewenstein, 2018). It is

66 a major driver of learning and determinative of the success of development; it applies to humans

67 and to non-human animals (ibid.). Recent work has begun to demonstrate its preserved features

68 across species and over the lifespan. It appears to be associated with at least somewhat discrete

69 neural circuits (Gruber et al., 2014; Lau et al., 2020; Cervera et al., 2019). While there is no

70 universally accepted definition of the term, curiosity, a common feature of most definitions is

71 that it involves a demand for information that is intrinsic non-strategic, and/or non-instrumental.

72 This definition distinguishes curiosity from information seeking in, for example, the well- 
73 understood bandit task (Cohen et al., 2007). In that case, information-seeking, and subsequent

74 learning, is driven by rewards.

75

\section{Latent learning}

Classical concepts of learning held that all learning is driven by reinforcement

contingencies. Formally, the presence or lack of reward or punishment promotes the positive or negative associations between actions and the results they produce, or between different stimuli. These ideas are fundamental to the "Law of Effect" and are fundamental to the idea of Hebbian learning (Thorndike, 1927; Walton et al., 2010). That work, in elaborated form, is central to reinforcement learning, one of most successful psychological theories and the basis of a generation of systems neuroscience. Notably, these concepts in turn form the basis of much of modern machine learning.

However, even as early as 1929, it was shown by Hugh C. Blodgett, a graduate student of Edward Tolman at UC Berkeley, that robust learning could occur in the absence of reward (Blodgett, 1929). This makes sense to anyone who can still effortlessly recite the lyrics to advertising jingles from their childhood. But it was the source of much debate in psychology departments in the first half of the 20th century. Later members of Tolman's lab followed up on this work, which is summarized in Tolman's classic paper (Tolman, 1948). In this paper, Tolman characterized the simpler reinforcement learning approach as the "stimulus response school," and described the idea of cognitive maps, which he believed depended on latent learning.

In a classic latent learning setup, a rat is released into a large maze with no reward. Naive rats typically amble around the maze, ostensibly with no purpose. According to behaviorist theories of the stimulus-response school, the rat should not learn anything in particular about the

96 layout of the maze because no reward or punisher was provided. Later, in a different 
97 experimental session, the experimenters can introduce a reward to a specific location in the

98 maze. The same rats, on encountering the reward, would then be given a chance to run the maze

99 again from the beginning. The rats with maze exposure learned to locate the reward much more

100 quickly than ones who were naive to that maze. Tolman coined the term "latent learning" to

101 describe the learning of maze layout by rats when they were not reward motivated. To drive this

102 point home, a later experiment provided food and water in the maze and rats went through latent

103 learning when they are satiated (not motivated). They showed high efficiency using the learned

104 maze layout to find food or water when later they visited the maze hungry or thirsty (Tolman,

105 1948; Spence et al., 1950). Tolman proposed that these rats had learned (latently) the layout of

106 the maze without any stimulus-reward association, a theory that violated then-dominant

107 dogmatic versions of behaviorism. Specifically, Tolman proposed that latent learning allowed

108 the rats to form a cognitive map of the layout of the maze.

\section{Curiosity and cognitive maps}

111 Blodgett's rats did not move randomly throughout the maze for no reason. Their decision

112 to move was not costless, nor was the mental effort involved in observing and consolidating

113 those observations into a mental map. Any forager placed within a complex natural environment

114 must naturally trade off between the costs and benefits of exploration. In addition to the

115 metabolic costs of locomotion, sensory processing, and learning, active exploration carries

116 opportunity costs: that time could be better spent searching for food, courting and reproducing,

117 or avoiding predators. The decision-maker, subject inevitably to extremely high selection

118 pressures, does not wander idly. But we also can use any standard extrinsically rewarded

119 economic explanation to motivate latent learning. Even motivational processes driven by distal 
120 reward seeking must necessarily discount future rewards and uncertain rewards, and the benefits

121 of exploration are unavoidably delayed beyond the temporal horizon and, individually,

122 infinitesimally unlikely. So reward-maximizing calculation is unlikely to motivate search.

123 Evolution must step in and endow the decision-maker with intrinsic motivation to learn (Bennett

124 et al., 2016; Grant et al., 1998; Tversky and Edwards, 1966; Kidd and Hayden, 2015).

125 Curiosity is likely to be especially important for learning cognitive maps. Cognitive maps

126 refer to detailed mental representations of the relationship between various elements in the world

127 and their sequelae. Having a cognitive map allow a decision-maker to not just guess what will

128 happen but also to deal with unexpected changes in our environment. The classic idea about

129 cognitive maps - also attributable to Tolman - is that they allow us to respond flexibly when the

130 layout of a maze was later changed (Tolman, 1948; Tolman et al., 1946). That kind of flexibility

131 - the ability to have insight - is very difficult to implement with basic reinforcement learning

132 processes (Schoenbaum and Roesch, 2005; Wilson et al., 2014). Instead, we must have a

133 sophisticated representation of the structure of the world, and the ability to navigate

134 counterfactuals and unexpected changes.

135 Cognitive maps require a level of detail that is not normally available from reinforcement

136 learning processes. Specifically, cognitive maps involve a detailed representation of the linkages

137 between adjacent spaces, and allow for vicarious travel along those linkages. That representation,

138 then, is a level of abstraction higher than the simple stimulus-outcome-action mapping that

139 standard reinforcement learning gives. But because it is so much richer, and more detailed, it

140 requires orders of magnitude more information than standard reward-motivated reinforcement

141 learning can give. Getting that information cannot occur if it needs extrinsic rewards - those

142 rewards simply are not available in the environment. It requires intrinsic rewards. 
We propose that what Tolman observed as latent learning, was motivated and enabled by

144 curiosity and information-seeking behavior (free, non-motivated exploration in his case).

145 However, Tolman conceived of latent learning as a fundamentally passive process, one that took

146 place during apparently purposeless exploration - almost as if by accident. We propose, instead,

147 that latent learning in practice tends to be more actively driven. However, this purposive

148 exploration must be e driven by the evolutionary advantage brought by curiosity and ultimately

149 by the extreme information gap experienced by foragers in the natural world.

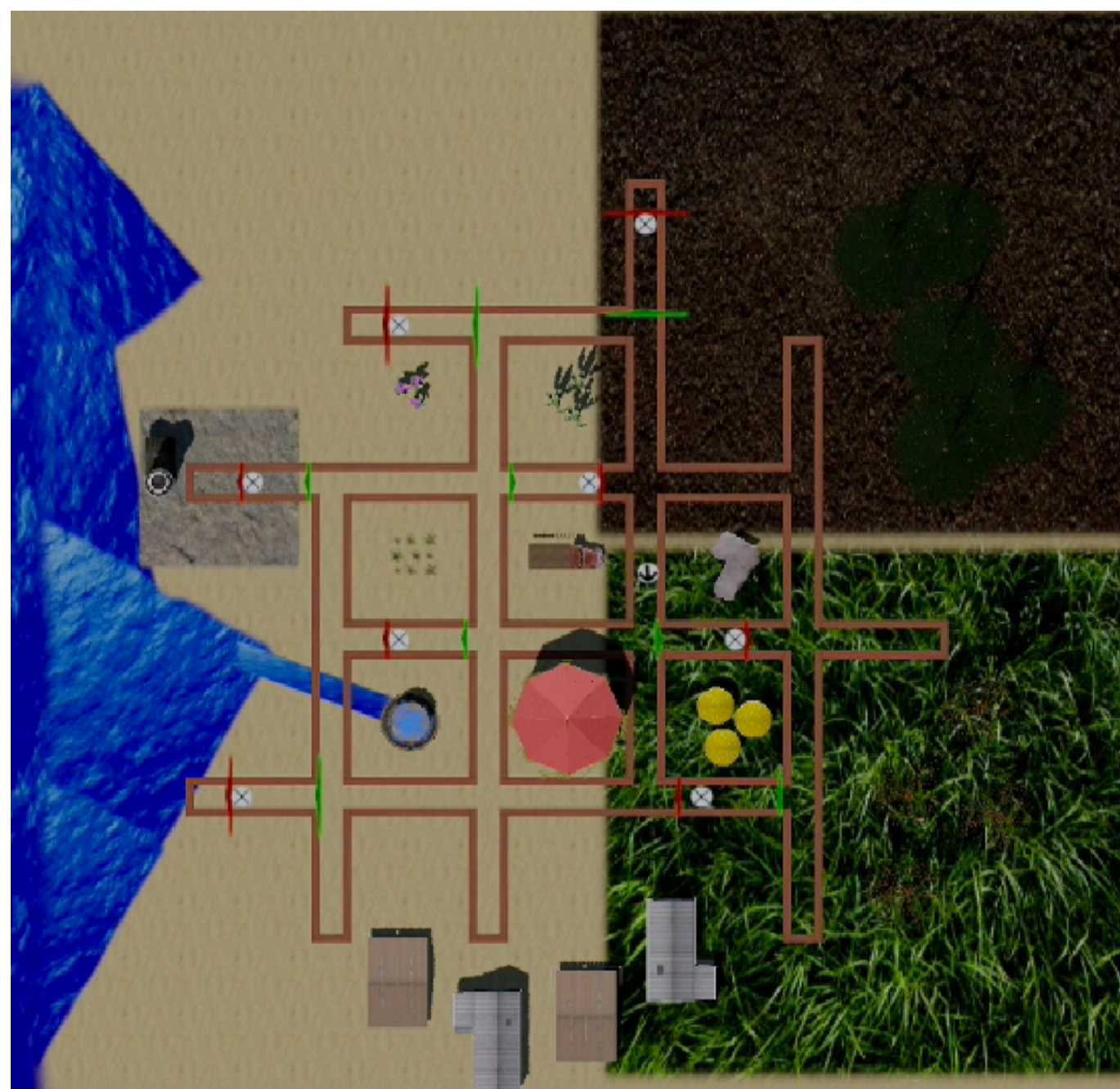

Figure 1. A virtual maze our lab has used for monkeys based on the classic 14-unit T- 
154 maze cannot be expanded into a linear track in contrast to the original maze. Tolman

155 and his graduate students placed rats in mazes like this one and found that they would

156 explore the maze unrewarded and would demonstrably learn the features of the

157 structure of the maze in the absence of rewards, a result that is difficult to explain using

158 then-dominant simple stimulus-response learning theories. Tolman proposed that the

159 rats generated a cognitive map that instantiated features of the maze and could be

160 consulted to drive flexible behavior.

161

162

\section{The analogy to artificial intelligence}

164 to the problems faced by artificially intelligent (AI) agents in machine learning problems.

165 Consider for example that AI can be trained to perform a classic Atari games using

166 straightforward RL principles (Mnih et al., 2013). In these cases, the agent must learn a strategy

167 using an elaborated gradient descent procedure - not different from how the stimulus-response

168 school imagined rats learned a maze. But those games, especially the ones that AI is good at, like

169 Video Pinball and Breakout, differ from natural situations in key ways that make them easy for

170 RL algorithms. In contrast, the real world - and some games like Pitfall and Montezuma's

171 Revenge - are what is known as hard-exploration problems (Bellemare et al., 2016; Ecoffet et al.,

172 2018; Ecoffet et al., 2019). In such problems, rewards are sparse (they require dozens or

173 hundreds of correct actions), so gradient descent procedures are nearly useless. For example, in

174 Pitfall, the first opportunity to gain any points comes after $\sim 60$ seconds of perfect play involving

175 dozens of precisely timed moves. Moreover, rewards are often deceptive (they result in highly

176 suboptimal local minima, so getting a small reward promotes adherence to a suboptimal 
177 strategy). Finally, real-world problems often present very abstract goals (e.g. "design an

178 attractive furniture layout for the study on a medium budget"). RL agents that do well at

179 relatively naturalistic hard-exploration games tend to have deliberate hard-coded exploration

180 bonuses.

The AI domain provides a good illustration of how cognitive maps can be crucial for the

182 success of curiosity. The optimal search strategy in sparse (natural) environments is typically to

183 identify a locally promising region and then perform strategic explorations from that spot to

184 identify subsequent ones (Ecoffet et al., 2020). That exploration will not be random, but will take

185 place along identified high-value destinations. AI agents suffer from the problem of detachment,

186 that is, when they explore the environment, they leave the relatively high-reward areas of space

187 to explore lower-reward ones. Most such areas are likely to be dead ends, and, when a dead end

188 is detected, the agent ought to return to the high reward area and pursue other promising paths.

189 However, the basic curiosity-based approach, which gives intrinsic rewards for novelty, repel the

190 agent from returning to the promising region of space, precisely because it's the most familiar

191 and least intrinsically rewarded (it's also not extrinsically rewarding, because any extrinsic

192 reward has been consumed on the path there, and does not replenish in the meantime). This in

193 turn requires making some kind of internal map of space so that the agent can return to the locus

194 of high potential reward and explore more efficiently than a wholly random path. One example

195 would be a bee hunting for promising hive sites. That bee must be able to have some map of the

196 local environment (Cheeseman et al., 2014).

197 A closely related problem that AI agents - and real-world agents as well - face, is the

198 problem of derailment. To explore a space efficiently, an agent must be able to return to

199 promising states and use those as a starting point for efficient exploration. From there, the agent 
must engage in random search. However, in real environments, returning to a promising state

201 may require a very precise sequence of actions that cannot be deviated from - so stochasticity

202 must be controlled until that state is achieved, at which case it must begin again in earnest. As

203 such, stochastic search must be carefully controlled depending on one's place in the larger

204 environment - which requires basic mapping functions, and cannot be done with simple RL-type

205 learning. Moreover, important factors governing the exploration process, such as detecting an

206 information gap, deriving the value of information itself, and directing exploration towards

207 potential sites that might be low in external reward but high in information/entropy, simply

208 cannot be supported by only experienced reward history. The key to achieve this is to have a

209 mental map, or internal model, of what is available, and what is novel and potentially offer high

210 information content (high entropy).

211 An intuitive example is a monkey learning to harvest fruit at the top of a tree. The tree is

212 tall and with lush leaves such that the fruits are not visible from the safety on the ground. While

213 monkeys need the calories and nutrients to survive and thrive, and the fruits offer both, the climb

214 is treacherous and the cost of falling is high. So in order to maximize reward and minimize cost,

215 but also being able to make better and better predictions on which new branches to go to for

216 potentially more fruits and/or a better view to detect them, the monkey must have some kind of

217 map of its internal state in order to strategically deploy search. 


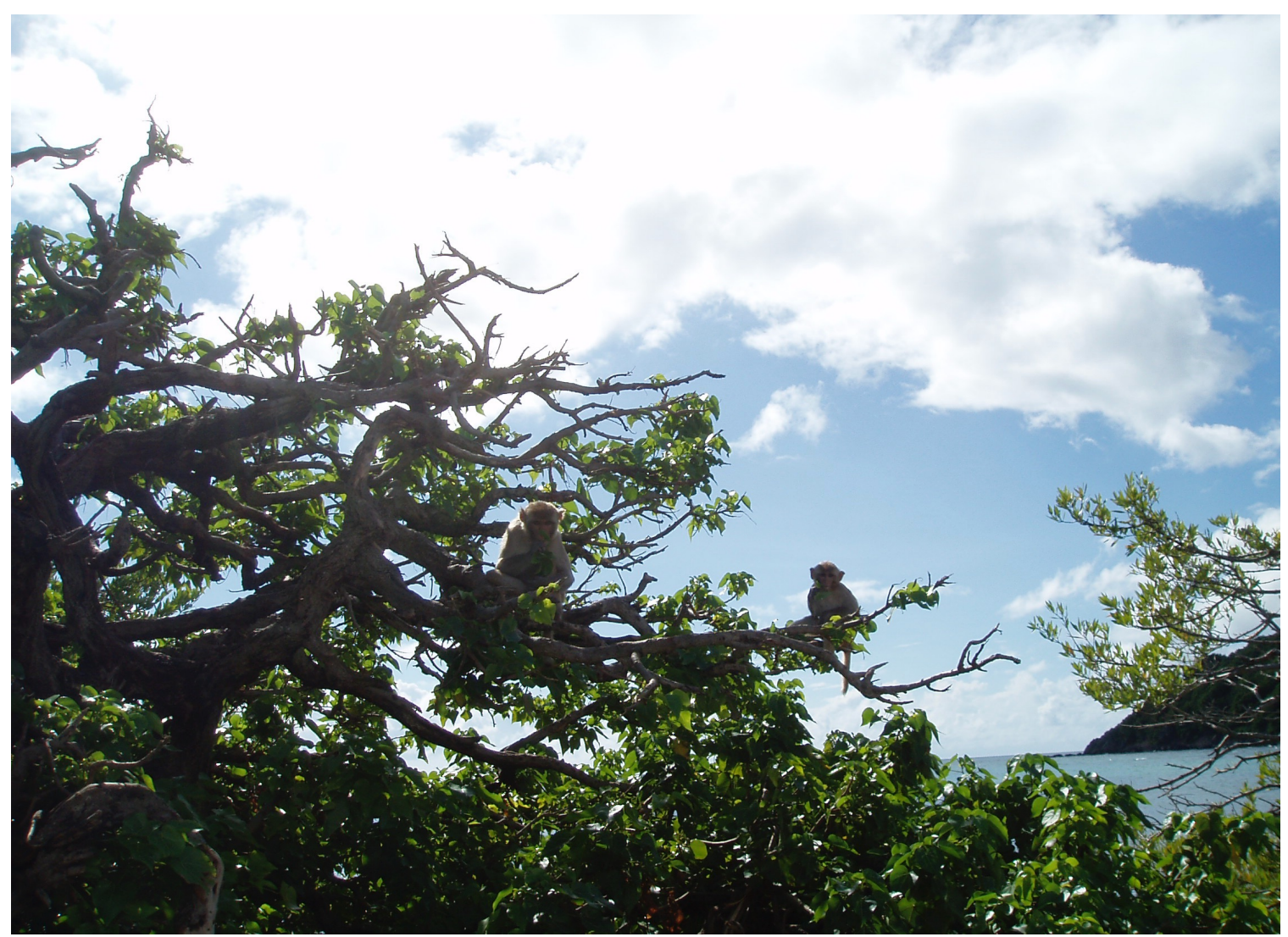

Figure 2. Monkey in a tree, illustrating the problem of derailment in curiosity research. The monkey must learn foraging strategy through trial and error, which requires highly variable exploration of the environment. But getting to the end of a branch is somewhat risky and requires suppressing stochastic variability. To successfully deploy curiosity the monkey must have a cognitive map of where variability is good and where it is bad.

\section{Operational definition of curiosity}

Developing these ideas about curiosity, latent learning, and cognitive maps holds great

230 potential in neuroscience. However, it faces several problems from the get-go. We and others

231 have defined curiosity as a motivation to seek information that lacks instrumental or strategic

232 benefit (Kidd and Hayden, 2015; Loewenstein, 1994; Berlyne, 1966; Oudeyer et al., 2016). By

233 this definition, many explorative and playing behaviors qualify as a demonstration of curiosity

234 (Glickman and Sroges, 1966; Byrne, 2013). From the perspective of scientific research, this

235 definition is problematic because it is vague and so does not readily lend itself to the laboratory. 
236 In an effort to remedy these drawbacks, we developed an operational definition that combines

237 three criteria: (1) a curious research subject is willing to sacrifice primary reward in order to

238 obtain information; (2) the amount of reward a subject is willing to pay scales with the amount of

239 potentially available additional information; and (3) additionally gained information provides no

240 obvious instrumental or strategic benefit. This definition is deliberately conservative; that is, it

241 excludes much of behavior that is likely to be curiosity-driven.

242

This definition justifies the observing task as a laboratory instrument for manipulating

243 curiosity, although that task is subject to certain criticisms that have not yet been resolved (e.g.

244 Beierholm and Dayan, 2010). On the other hand, formalized risky choice tasks offer a

245 straightforward opportunity to understand preferences with close control of information

246 (Heilbronner, 2017; Heilbronner and Hayden, 2016). We therefore devised a more complex task

247 that would circumvent published criticisms of the observing task (Wang and Hayden, 2019).

248 This task is based on the observation that monkeys seek counterfactual information - information

249 about what would have happened had they chosen differently. In the counterfactual curiosity

250 task, monkeys choose between two risky offers. Behavior is overtrained (hundreds of thousands

251 of trials), reducing the chance that monkeys have erroneous theories about payoff distributions.

252 During testing, monkeys are sometimes given the opportunity to choose an option that will

253 provide valid information about the outcome that would have occurred had they chosen the other

254 option. Monkeys are willing to pay to choose this option, indicating that they are curious about

255 counterfactual outcomes. Moreover, monkeys paid more for options that provide more

256 counterfactual information. We speculate that this curiosity-driven information-seeking helps

257 monkeys to develop a sophisticated cognitive map of their task environments. 

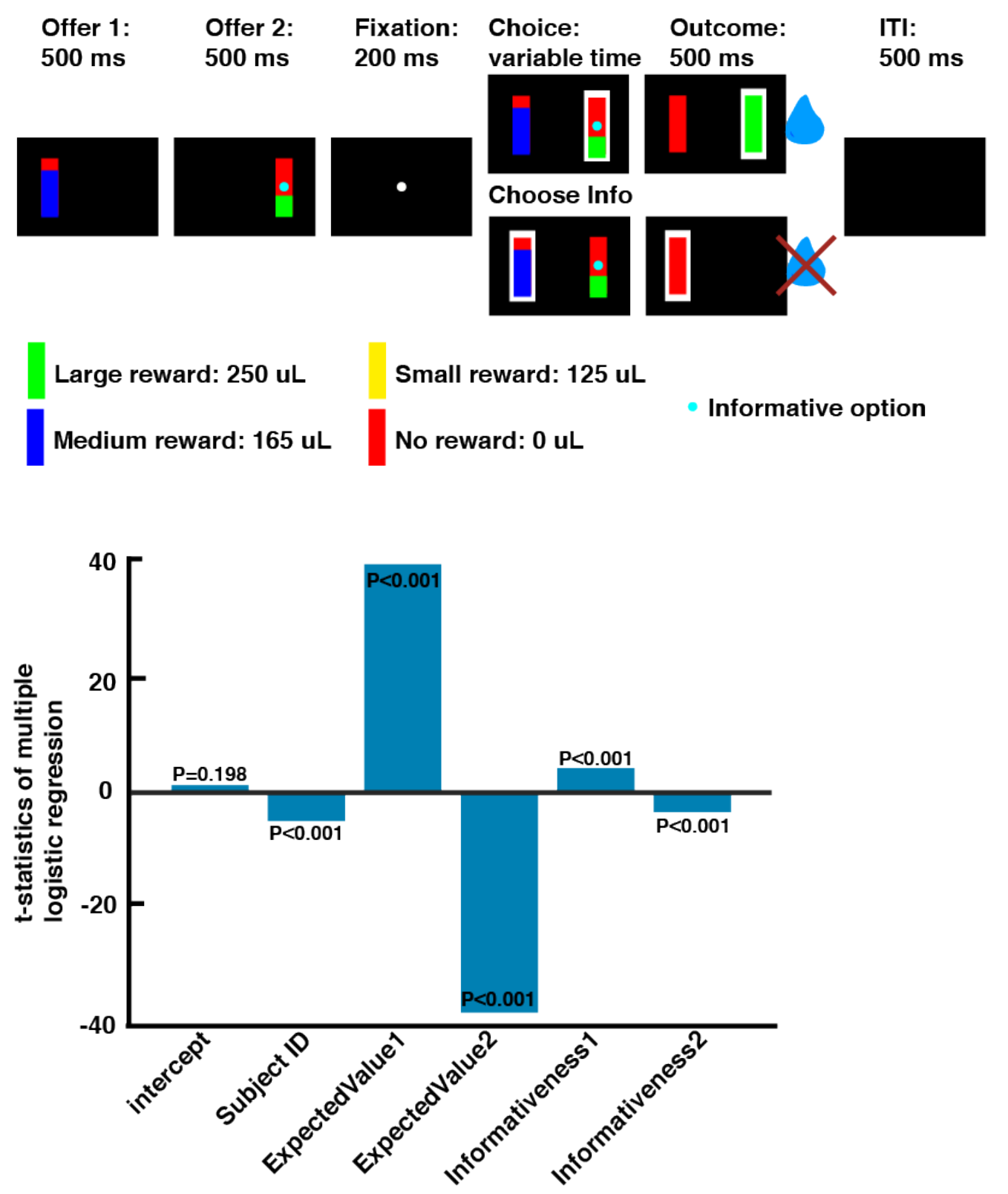

Figure 3. In our curiosity task, subjects could choose between risky options for juice rewards. In some trials, they could also gain information about what would have occurred had they chosen differently. By analyzing preference curves on such trials, we could quantify their subjective value of counterfactual information. We found a small but significant positive valuation of counterfactual information in both subjects tested.

\section{Functional neuroanatomy of curiosity in the frontal lobes}

Our ultimate goal is to understand the neural circuitry underlying curiosity-driven choice. Here we summarize the tentative picture, with a focus on two prefrontal regions, the orbitofrontal 
cortex (OFC) and the dorsal anterior cingulate cortex (dACC). Both regions are implicated in neuroimaging studies of curiosity (Charpentier et al., 2018, Kobayashi and Hsu, 2019; Van

271 Lieshout et al., 2018). The neuroanatomy of curiosity is more complex and includes other areas

272 such as hippocampal areas (Kang et al., 2009; Gruber et al., 2014; Jepma et al., 2012) and basal

273 ganglia (White et al., 2019). But we would like to highlight OFC and dACC for their potential

274 involvement that bridges curiosity, latent learning, and cognitive maps. Briefly, we propose that

275 the OFC and AACC have complementary roles and that these roles are best described as

276 acquisition and update of the cognitive maps and instrumental application of cognitive maps,

277 respectively.

278

Orbitofrontal cortex: We propose that OFC serves to track the innate value of

280 information, to maintain a cognitive map of state space, and to update that map when new

281 information is gained. The clear role of OFC in cognitive mappings has been one of the major

282 intellectual advances of the past decade, and is demonstrated in rodent, monkey, and human

283 species. (Wilson et al., 2014; Wang and Hayden 2017; Wikenheiser and Schoenbaum, 2016;

284 Schuck and Niv, 2016). OFC also carries various contextual variables not directly related to

285 economics (Roesch et al., 2006; Feierstein et al., 2006; Wallis et al., 2001; Sleezer et al., 2016).

286 Our lab's contribution to this comes from a study using a variant of the observing task

287 (Blanchard et al., 2015). We found that OFC neurons encoded the value of information and

288 (confirming much previous work) the value of offers. Critically, however, OFC used distinct

289 codes for informational value and for more standard juice value - highlighting the special role of

290 information - it was not an additive value to the primary reward, but of its own distinct category.

291 this distinction is possibly for completing and updating the cognitive map of the 
292

293

294 but rather, represents task-relevant information in multiple formats, as would be expected in a

295 map rather than a simple reinforcement learning situation. Of course, OFC does not achieve this

296 alone. Studies using similar paradigms discovered that information is signaled by other systems,

297 including the midbrain dopamine system (Bromberg-Martin and Hikosaka, 2009 and 2011;Guru

298 et al., 2020).

299

300

301

302

303

304

305

306

307

308

task/environmental structure -- even though this information may not offer strategic benefit in any near future. In other words, OFC doesn't use a single coherent value code across contexts,

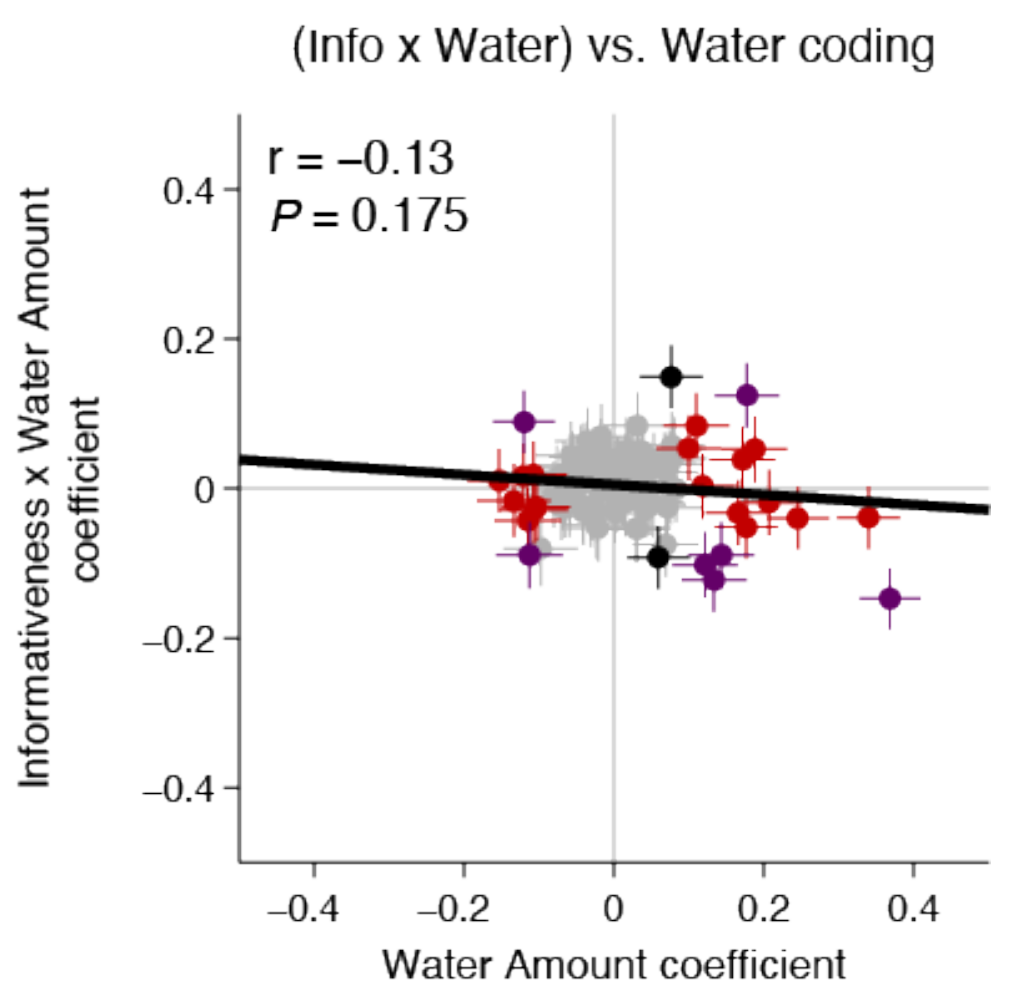

Figure 4. In our observing task, responses of an ensemble of OFC neurons to offers varying in informational value and reward value. We find that individual neurons encode both variables (horizontal and vertical axes indicate tuning coefficients for the two dimensions respectively). However, those codes are themselves uncorrelated, as indicated by the lack of a significant slope between the two dimensions.

ACC: regulation of information flow in the cognitive map 
We propose that dACC plays a distinct and complementary role of OFC. Specifically, it

310 appears to track both information delivery and level and task demands for use by OFC in

311 updating the cognitive map and applying it to instrumental use. This idea is motivated by the

312 observation that dACC tracks informativeness, counterfactual information (White et al., 2019,

313 Hayden et al., 2009), environmental demands (Kolling et al., 2012; Hayden et al., 2011), as well

314 as various economic variables (e.g. Bush et al., 2002; Seo and Lee, 2007; Azab and Hayden,

$3152017,2018)$. It is further motivated by observations about the relative hierarchical positions of

316 the two regions and the relative contributions to choice (Rushworth et al., 2011; Hunt et al.,

317 2018; Yoo and Hayden, 2018).

318 In a recent study using a Pavlovian paradigm, White et., al. (2019) trained monkeys to

319 associate juice rewards with various reward probabilities with different fractals. Then,

320 information (or non-information) stimuli followed the fractals. Single units in dACC showed

321 increased firing rates to increased uncertainty, and thus to higher expectation of information

322 (when the uncertainty resolved). Moreover, dACC firing rates ramped up to the anticipation of

323 the information that came with the resolution of the uncertainty. In other words, dACC neurons

324 did not simply encode different levels of uncertainty which remained at a constant level for each

325 trial; nor did they ramp up firing rates in anticipation to reward delivery These results showed

326 that dACC tracks the lack of information and anticipates the delivery of information. Our own

327 results paint a similar picture (Wang and Hayden, 2020). Using the observing task, we find that,

328 following uninformative choices, firing is enhanced until information delivery and is scaled with

329 the amount of information gained through resolving gamble uncertainty. Moreover, in our study,

330 enhanced dACC firing also correlated with higher probability of information-seeking choices

331 later in the trial. 


\section{Conclusion and future directions}

334 Curiosity is a strong motivator of learning, development, and choice. It has long been

335 treated mystically, as if it is impenetrable to scholarly study. This attitude is found, for example,

336 in some of the research that treats curiosity as a human-specific phenomenon. Even when treated

337 as a regular psychological phenomenon, curiosity is often studied in an ad hoc manner. That

338 approach was necessary for early studies, but more recent work has made great progress in

339 developing formal approaches to understand the phenomenon systemically study its neural

340 substrates. That formal approach, aided by remarkable progress in AI, has in turn allowed

341 neuroscientists to tentatively start to understand the circuity of curiosity. That work in turn will

342 likely be critical for understanding naturalistic decision-making, which is marked by the need to

343 make quick decisions with orders of magnitude less information than would be idea. 
METHODS

\section{General Methods}

All animal procedures were performed at the University of Rochester (Rochester, NY,

348 USA) and were approved by the University of Rochester Animal Care and Use Committee. All

349 experiments were conducted in compliance with the Public Health Service's Guide for the Care

350 and Use of Animals. Two male rhesus macaques (Macaca mulatta), aged 9-10 years and

351 weighting 8.0-9.9 kg served as subjects. Both subjects had extensive previous experience in risky

352 decision-making tasks. Subjects had full access to food (LabDiet 5045, ad libitum) while in their

353 home cages. Subjects received at minimum $20 \mathrm{~mL}$ per $\mathrm{kg}$ of water per day, although in practice

354 they received close to double this amount in the lab as a result of our experiments. No subjects

355 were sacrificed or harmed in the course of these experiments.

356 Visual stimuli were colored rectangles on a computer monitor (see Figure 1). Stimuli

357 were controlled by Matlab with Psychtoolbox. Eye positions were measured with Eyelink

358 Toolbox. A solenoid valve controlled the delivery duration of fluid rewards. Eye positions were

359 sampled at $1,000 \mathrm{~Hz}$ by an infrared eye-monitoring camera system (SR Research, Osgoode, ON,

360 Canada). A small mount was used to facilitate maintenance of head position during performance.

361 Subjects had never previously been exposed to decision-making tasks in which

362 counterfactual information was available.

363

\section{The Counterfactual Information Task}

The task structure was a close variant of a general one that we have used many times in

366 the past. Subjects fixated, in sequence, on two options, located on the two sides of the computer 
monitor. They had been extensively trained ( $5+$ years in both cases) on tasks like this in the past and were adept at making effective choices in those. counterfactual information (Fig. 1a). Due to the extensive exposure to similar tasks and the

371 simplicity of the current task, no pre-training was used. Both subjects were trained directly on

372 the current task and achieved above $80 \%$ accuracy within the first three days of training.

373 Following completion of additional training, we collected 8142 trials of behavior from both

374 subjects (5086 trials from subject B and 3056 trials from subject J). On each trial, subjects chose

375 between two randomly selected gambles presented asynchronously on the left and the right side

376 of the screen. Gambles were represented by rectangular visual stimuli and differed in three

377 dimensions: payoff, probability, and informativeness. Payoff came in three sizes, small (125

378 microliters), medium (165 microliters), and large (250 microliters), each corresponding to a

379 yellow, blue, and green portion of the rectangle, respectively. Probabilities were randomly drawn

380 from a uniform distribution between 0 and 1 (101 steps; step size 0.01 ). The height of the

381 yellow/blue/green portion of the rectangle indicated the probability of winning the gamble and

382 the height of the red portion indicated the probability of losing (that is receiving no reward for

383 that trial). Informativeness of a gamble was indicated by a cyan dot on the center of the rectangle

384 for an informative option and the lack of a cyan dot for a non-informative one. The informative

385 option promised valid information about the payoff that would have occurred had the alternative

386 option been chosen. Probability, payoff, and informativeness were independently randomized on

387 each trial. On $50 \%$ of the trials, only one option was informative (info choice trials). On $25 \%$ of

388 the trials, both options were informative (forced info trials). The forced info trials were

389 equivalent to what would be called full-feedback trials in human judgment and decision-making 
literature (Camilleri \& Newell, 2011). On the remaining 25\%, neither option was informative (no info trials). These are equivalent to what are called partial-feedback trials. manipulation) to probe macaques' preferences for uncertainty. Critically, via controls, we have

394 demonstrated that macaques treat these stimuli as if they provide explicit information about the 395 structures of gambles. delay. Offer 1 position was randomized for each trial. Then offer 2 appeared on the other side of the screen $(500 \mathrm{~ms})$ followed by another $500 \mathrm{~ms}$ delay. After a $200 \mathrm{~ms}$ fixation, both gambles appeared on the screen and subjects chose the preferred option by shifting gaze to it and maintaining that gaze for $200 \mathrm{msec}$. Subsequently, if an informative option was chosen, gamble

401 outcomes for both offers were resolved. If a non-informative option was chosen, the gamble

402 outcome for only the chosen offer was resolved. Resolution of a gamble involved filling the 403 gamble rectangle with the payoff color while delivering a water reward (if the gamble result was 404 win), or filling the gamble rectangle with red color and delivering no reward, (if the gamble 405 result was a loss). The outcome epoch lasted for $800 \mathrm{~ms}$ and was followed by a $1000 \mathrm{~ms}$ intertrial interval (ITI) and then the start of next trial.

407 Consider, for example, a subject performing the following trial. First, offer 1 appears on 408 the left side of the computer monitor. It is a non-informative option (it has no cyan dot) with $40980 \%$ probability (indicated by the height of the blue section) of yielding a medium reward (165 $410 \mathrm{uL}$, indicated by blue color) and 20\% probability of yielding no reward (indicated by red color).

411 After a second, offer 2 appears. Offer 2 is an informative option (it has a cyan dot) that 412 corresponds to a $45 \%$ probability (indicated by height of green segment) of yielding $250 \mathrm{uL}$ 
413 (indicated by green color), and 55\% probability of getting no reward (indicated by red color).

414 After a brief pre-choice eye fixation, the subject chooses offer 2 . This choice resulted in a win

415 with water reward delivery and the presentation of counterfactual outcome information.

416 


\section{REFERENCES}

Azab, H., \& Hayden, B. Y. (2017). Correlates of decisional dynamics in the dorsal anterior cingulate cortex. PLoS biology, 15(11), e2003091.

Azab, H., \& Hayden, B. Y. (2018). Correlates of economic decisions in the dorsal and subgenual anterior cingulate cortices. European Journal of Neuroscience, 47(8), 979-993.

Beierholm, U. R., \& Dayan, P. (2010). Pavlovian-instrumental interaction in 'observing behavior'. PLoS computational biology, 6(9).

Bellemare, M., Srinivasan, S., Ostrovski, G., Schaul, T., Saxton, D., \& Munos, R. (2016). Unifying countbased exploration and intrinsic motivation. In Advances in neural information processing systems (pp. 1471-1479).

Bennett, D., Bode, S., Brydevall, M., Warren, H., \& Murawski, C. (2016). Intrinsic valuation of information in decision making under uncertainty. PLoS computational biology, 12(7).

Berlyne, D. E. (1966). Curiosity and exploration. Science, 153(3731), 25-33.

Blanchard, T. C., Hayden, B. Y., \& Bromberg-Martin, E. S. (2015). Orbitofrontal cortex uses distinct codes for different choice attributes in decisions motivated by curiosity. Neuron, 85(3), 602-614.

Blodgett, H. C. (1929). The effect of the introduction of reward upon the maze performance of rats. University of California publications in psychology.

Bromberg-Martin, E. S., \& Hikosaka, O. (2011). Lateral habenula neurons signal errors in the prediction of reward information. Nature neuroscience, 14(9), 1209.

Bromberg-Martin, E. S., \& Hikosaka, O. (2009). Midbrain dopamine neurons signal preference for advance information about upcoming rewards. Neuron, 63(1), 119-126.

Bush, G., Vogt, B. A., Holmes, J., Dale, A. M., Greve, D., Jenike, M. A., \& Rosen, B. R. (2002). Dorsal anterior cingulate cortex: a role in reward-based decision making. Proceedings of the National Academy of Sciences, 99(1), 523-528.

Byrne, R. W. (2013). Animal curiosity. Current Biology, 23(11), R469-R470.

Calhoun, A. J., \& Hayden, B. Y. (2015). The foraging brain. Current Opinion in Behavioral Sciences, 5, 24-31.

Cervera, R. L., Wang, M. Z., \& Hayden, B. (2020). Curiosity from the Perspective of Systems Neuroscience. PsychArxiv.

Charpentier, C. J., Bromberg-Martin, E. S., \& Sharot, T. (2018). Valuation of knowledge and ignorance in mesolimbic reward circuitry. Proceedings of the National Academy of Sciences, 115(31), E7255-E7264.

Cheeseman, J. F., Millar, C. D., Greggers, U., Lehmann, K., Pawley, M. D., Gallistel, C. R., ... \& Menzel, R. (2014). Way-finding in displaced clock-shifted bees proves bees use a cognitive map. Proceedings of the National Academy of Sciences, 111(24), 8949-8954.

Cohen, J. D., McClure, S. M., \& Yu, A. J. (2007). Should I stay or should I go? How the human brain manages the trade-off between exploitation and exploration. Philosophical Transactions of the Royal Society B: Biological Sciences, 362(1481), 933-942. 
472

473

474

475

476

477

478

479

480

481

482

483

484

485

486

487

488

489

490

491

492

493

494

495

496

497

498

499

500

501

502

503

504

505

506

507

508

509

510

511

512

513

514

515

516

517

518

519

520

521

522

523

524

525

526

David, S. V., \& Hayden, B. Y. (2012). Neurotree: A collaborative, graphical database of the academic genealogy of neuroscience. PloS one, $7(10)$.

Ecoffet, A., Huizinga, J., Lehman, J., Stanley, K. O., \& Clune, J. (2019). Go-explore: a new approach for hard-exploration problems. arXiv preprint arXiv:1901.10995.

Ecoffet, A., Huizinga, J., Lehman, J., Stanley, K. O., \& Clune, J. (2020). First return then explore. arXiv preprint arXiv:2004.12919.

Ecoffet, A., Huizinga, J., Lehman, J., Stanley, K. O., \& Clune, J. (2018). Montezuma's revenge solved by go-explore, a new algorithm for hard-exploration problems (sets records on pitfall, too). Uber Engineering Blog, Nov.

Elliott, M. H. (1928). The effect of change of reward on the maze performance of rats. University of California Press.

Farashahi, S., Azab, H., Hayden, B., \& Soltani, A. (2018). On the flexibility of basic risk attitudes in monkeys. Journal of Neuroscience, 38(18), 4383-4398.

Feierstein, C. E., Quirk, M. C., Uchida, N., Sosulski, D. L., \& Mainen, Z. F. (2006). Representation of spatial goals in rat orbitofrontal cortex. Neuron, 51(4), 495-507.

Glickman, S. E., \& Sroges, R. W. (1966). Curiosity in zoo animals. Behaviour, 26(1-2), 151-187.

Golman, R., \& Loewenstein, G. (2018). Information gaps: A theory of preferences regarding the presence and absence of information. Decision, 5(3), 143.

Gottlieb, J., \& Oudeyer, P. Y. (2018). Towards a neuroscience of active sampling and curiosity. Nature Reviews Neuroscience, 19(12), 758-770.

Grant, S., Kajii, A., \& Polak, B. (1998). Intrinsic preference for information. Journal of Economic Theory, 83(2), 233-259.

Gruber, M. J., Gelman, B. D., \& Ranganath, C. (2014). States of curiosity modulate hippocampusdependent learning via the dopaminergic circuit. Neuron, 84(2), 486-496.

Guru, A., Seo, C., Post, R. J., Kullakanda, D. S., Schaffer, J. A., \& Warden, M. R. (2020). Ramping activity in midbrain dopamine neurons signifies the use of a cognitive map. bioRxiv.

Hayden, B. Y., Pearson, J. M., \& Platt, M. L. (2011). Neuronal basis of sequential foraging decisions in a patchy environment. Nature neuroscience, 14(7), 933.

Hayden, B. Y., Pearson, J. M., \& Platt, M. L. (2009). Fictive reward signals in the anterior cingulate cortex. science, 324(5929), 948-950.

Hayden, B. Y., Heilbronner, S. R., Pearson, J. M., \& Platt, M. L. (2011). Surprise signals in anterior cingulate cortex: neuronal encoding of unsigned reward prediction errors driving adjustment in behavior. Journal of Neuroscience, 31(11), 4178-4187.

Heilbronner, S. R., \& Hayden, B. Y. (2016). The description-experience gap in risky choice in nonhuman primates. Psychonomic bulletin \& review, 23(2), 593-600.

Hayden, B. Y., \& Platt, M. L. (2010). Neurons in anterior cingulate cortex multiplex information about reward and action. Journal of Neuroscience, 30(9), 3339-3346. 
Heilbronner, S. R. (2017). Modeling risky decision-making in nonhuman animals: shared core features. Current opinion in behavioral sciences, 16, 23-29.

Hunt, L. T., Malalasekera, W. N., de Berker, A. O., Miranda, B., Farmer, S. F., Behrens, T. E., \& Kennerley, S. W. (2018). Triple dissociation of attention and decision computations across prefrontal cortex. Nature neuroscience, 21(10), 1471-1481.

Jepma, M., Verdonschot, R. G., Van Steenbergen, H., Rombouts, S. A., \& Nieuwenhuis, S. (2012). Neural mechanisms underlying the induction and relief of perceptual curiosity. Frontiers in behavioral neuroscience, 6,5 .

Kang, M. J., Hsu, M., Krajbich, I. M., Loewenstein, G., McClure, S. M., Wang, J. T. Y., \& Camerer, C. F. (2009). The wick in the candle of learning: Epistemic curiosity activates reward circuitry and enhances memory. Psychological science, 20(8), 963-973.

Kidd, C., \& Hayden, B. Y. (2015). The psychology and neuroscience of curiosity. Neuron, 88(3), 449-460.

Kobayashi, K., \& Hsu, M. (2019). Common neural code for reward and information value. Proceedings of the National Academy of Sciences, 116(26), 13061-13066.

Kolling, N., Behrens, T. E., Mars, R. B., \& Rushworth, M. F. (2012). Neural mechanisms of foraging. Science, 336(6077), 95-98.

Lau, J. K. L., Ozono, H., Kuratomi, K., Komiya, A., \& Murayama, K. (2020). Shared striatal activity in decisions to satisfy curiosity and hunger at the risk of electric shocks. Nature Human Behaviour, 1-13.

Loewenstein, G. (1994). The psychology of curiosity: A review and reinterpretation. Psychological bulletin, 116(1), 75.

Mnih, V., Kavukcuoglu, K., Silver, D., Graves, A., Antonoglou, I., Wierstra, D., \& Riedmiller, M. (2013). Playing atari with deep reinforcement learning. arXiv preprint arXiv:1312.5602.

Mobbs, D., Trimmer, P. C., Blumstein, D. T., \& Dayan, P. (2018). Foraging for foundations in decision neuroscience: Insights from ethology. Nature Reviews Neuroscience, 19(7), 419-427.

Oudeyer, P. Y., Gottlieb, J., \& Lopes, M. (2016). Intrinsic motivation, curiosity, and learning: Theory and applications in educational technologies. In Progress in brain research (Vol. 229, pp. 257-284). Elsevier.

Oudeyer, P. Y., \& Smith, L. B. (2016). How evolution may work through curiosity-driven developmental process. Topics in Cognitive Science, 8(2), 492-502.

Pirrone, A., Azab, H., Hayden, B. Y., Stafford, T., \& Marshall, J. A. (2018). Evidence for the speed-value trade-off: Human and monkey decision making is magnitude sensitive. Decision, 5(2), 129.

Roesch, M. R., Taylor, A. R., \& Schoenbaum, G. (2006). Encoding of time-discounted rewards in orbitofrontal cortex is independent of value representation. Neuron, 51(4), 509-520.

Rushworth, M. F., Noonan, M. P., Boorman, E. D., Walton, M. E., \& Behrens, T. E. (2011). Frontal cortex and reward-guided learning and decision-making. Neuron, 70(6), 1054-1069.

Schoenbaum, G., \& Roesch, M. (2005). Orbitofrontal cortex, associative learning, and expectancies. Neuron, 47(5), 633-636.

Schuck, N. W., Cai, M. B., Wilson, R. C., \& Niv, Y. (2016). Human orbitofrontal cortex represents a cognitive map of state space. Neuron, 91(6), 1402-1412. 
582

583

584

585

586

587

588

589

590

591

592

593

594

595

596

597

598

599

600

601

602

603

604

605

606

607

608

609

610

611

612

613

614

615

616

617

618

619

620

621

622

623

624

625

626

627

628

629

630

631

632

633

634

635

636

Seo, H., \& Lee, D. (2007). Temporal filtering of reward signals in the dorsal anterior cingulate cortex during a mixed-strategy game. Journal of neuroscience, 27(31), 8366-8377.

Sleezer, B. J., Castagno, M. D., \& Hayden, B. Y. (2016). Rule encoding in orbitofrontal cortex and striatum guides selection. Journal of Neuroscience, 36(44), 11223-11237.

Spence, K. W., Bergmann, G., \& Lippitt, R. (1950). A study of simple learning under irrelevant motivational-reward conditions. Journal of Experimental Psychology, 40(5), 539.

Strait, C. E., Blanchard, T. C., \& Hayden, B. Y. (2014). Reward value comparison via mutual inhibition in ventromedial prefrontal cortex. Neuron, 82(6), 1357-1366.

Thorndike, E. L. (1927). The law of effect. The American journal of psychology, 39(1/4), 212-222.

Tolman, E. C. (1948). Cognitive maps in rats and men. Psychological review, 55(4), 189.

Tolman, E. C., Ritchie, B. F., \& Kalish, D. (1946). Studies in spatial learning. I. Orientation and the shortcut. Journal of experimental psychology, 36(1), 13.

Tversky, A., \& Edwards, W. (1966). Information versus reward in binary choices. Journal of Experimental Psychology, 71(5), 680.

van Lieshout, L. L., Vandenbroucke, A. R., Müller, N. C., Cools, R., \& de Lange, F. P. (2018). Induction and relief of curiosity elicit parietal and frontal activity. Journal of Neuroscience, 38(10), 2579-2588.

Wallis, J. D., Anderson, K. C., \& Miller, E. K. (2001). Single neurons in prefrontal cortex encode abstract rules. Nature, 411(6840), 953-956.

Walton, M. E., Behrens, T. E., Buckley, M. J., Rudebeck, P. H., \& Rushworth, M. F. (2010). Separable learning systems in the macaque brain and the role of orbitofrontal cortex in contingent learning. Neuron, 65(6), 927-939.

Wang, M. Z., \& Hayden, B. Y. (2017). Reactivation of associative structure specific outcome responses during prospective evaluation in reward-based choices. Nature communications, 8(1), 1-13.

Wang, M. Z., \& Hayden, B. Y. (2019). Monkeys are curious about counterfactual outcomes. Cognition, 189, 1-10.

Wang, M. Z., \& Hayden, B. Y. (2020). Curiosity is associated with enhanced tonic firing in dorsal anterior cingulate cortex. bioRxiv. doi.org/10.1101/2020.05.25.115139

White, J. K., Bromberg-Martin, E. S., Heilbronner, S. R., Zhang, K., Pai, J., Haber, S. N., \& Monosov, I. E. (2019). A neural network for information seeking. Nature communications, 10(1), 1-19.

Wikenheiser, A. M., \& Schoenbaum, G. (2016). Over the river, through the woods: cognitive maps in the hippocampus and orbitofrontal cortex. Nature Reviews Neuroscience, 17(8), 513-523.

Wilson, R. C., Takahashi, Y. K., Schoenbaum, G., \& Niv, Y. (2014). Orbitofrontal cortex as a cognitive map of task space. Neuron, 81(2), 267-279.

Yoo, S. B. M., \& Hayden, B. Y. (2018). Economic choice as an untangling of options into actions. Neuron, 99(3), 434-447. 
bioRxiv preprint doi: https://doi.org/10.1101/2020.05.31.123380; this version posted June 1, 2020. The copyright holder for this preprint (which was not certified by peer review) is the author/funder. All rights reserved. No reuse allowed without permission. 